\title{
Are We Appropriately Testing for Vitamin B12 and Folate Deficiency?
}

\author{
Nawaal Davids ${ }^{1}$, Mariza Hoffmann ${ }^{1}{ }^{*}$, Nasheen Naidoo $^{1}$, Thandiwe Manjati ${ }^{2}$, Rajiv T Erasmus ${ }^{1}$
}

Affiliations:

${ }^{1}$ Division of Chemical Pathology, Department of

Pathology, University of Stellenbosch and National Health Laboratory Service (NHLS), Tygerberg Hospital, Cape Town, South Africa

2 Division of Haematology, Department of Pathology, University of Stellenbosch and National Health Laboratory Service (NHLS), Tygerberg Hospital, Cape Town, South Africa

\section{Correspondence to:}

\section{Mariza Hoffman}

Division of Chemical Pathology, Department of Pathology, University of Stellenbosch and National Health Laboratory Service (NHLS), Tygerberg

Hospital, Cape Town, South Africa

Email: mariza@sun.ac.za

How to cite this Article:

Davids N. Are we appropriately testing for vitamin B12 and folate deficiency? Ann. Clin. Chem. Lab. Med.2017:3(1); 8-14

DOI: http://dx.doi.org/10.3126/acclm.v3i1.15612

\section{(C) 2017 Nepalese Association for Clinical Chemistry}

This work is licensed under a Creative Commons Attribution-Share Alike 4.0 International License.

\section{Abstract \\ BACKGROUND}

The most common reason for assessing vitamin B12 and folate status is a clinical suspicion of deficiency along with the haematological abnormality of macrocytic anaemia. However, there is often a lack of a precise clinical or haematological picture to guide the appropriate investigation of these patients. Normal haemoglobin or mean cell volumes are often found, masking the need for appropriate investigation. When abnormal haematological parameters are found, it is often a sign of advanced deficiency. In this study we investigated whether patients with haematological findings of macrocytosis and/or anaemia are appropriately investigated for vitamin B12 and folate deficiencies and whether clinicians request metabolite screening to assist with the diagnosis.

\section{METHODS}

This was a retrospective audit of data obtained from the laboratory information system for a six month period at a tertiary academic hospital. Adult patients with macrocytosis, anaemia or both were selected and laboratory records reviewed to determine whether they were investigated for vitamin B12 and folate deficiency. RESULTS

Only $16.2 \%$ of patients with macrocytic anaemia, $7.8 \%$ of patients with isolated macrocytosis and $6.5 \%$ of patients with normocytic anaemia were tested for vitamin B12 and/or folate deficiency. Metabolite assays such as homocysteine and methylmalonic acid were not requested as part of a vitamin status assessment.

\section{CONCLUSION}

In our setting, vitamin B12 and folate assessment is a diagnostic dilemma, delaying identification of potentially debilitating disease. Clinicians need to be informed about earlier investigation and of the availability of metabolite screening and their use in establishing early deficiency.

KEY WORDS: Vitamin B12, folate, macrocytosis, anaemia, testing, metabolites. 


\section{INTRODUCTION}

Vitamin B12 (cobalamin) and folate are essential co-factors for important metabolic processes and their roles are co-dependent. Patients with vitamin B12 and/or folate deficiency present with macrocytic anaemia, however, haematological findings are a late sign of deficiency. ${ }^{1,2}$ Patients may present with isolated macrocytosis without anaemia, with normocytic anaemia without macrocytosis, or with the absence of both macrocytosis and anaemia, ${ }^{3}$ and in these cases it is necessary to determine whether deficiencies are appropriately investigated.

\section{Vitamin B12 and folate}

Vitamin B12 has important roles in nucleic acid synthesis and myelin production in the nervous system. Deficiencies occur due to a lack of nutritional intake, gastrointestinal mal absorption, autoimmune disease, severe hypothyroidism and inhibitors of gastric parietal cell function. ${ }^{2}$ Disturbances in vitamin B12 metabolism may also occur in Human Immunodeficiency Virus (HIV), pregnancy, diabetes mellitus, renal insufficiency, and multiple myeloma due to increased uptake by cells. ${ }^{4}$ Similarly, folate is involved in nucleic acid synthesis ${ }^{5}$ and is abundantly available in the diet. ${ }^{6}$ Deficiency is caused by mal absorptive states, conditions where there are increased folate demands (e.g. pregnancy, malignancy and haemolytic anaemia), chronic alcoholism, renal dialysis, anticonvulsant treatment or in HIV infection. ${ }^{67}$

\section{Haematological changes in vitamin B12 and folate deficiencies}

Vitamin B12 and folate deficiencies most commonly cause macrocytic anaemia (elevated mean cell volumes (MCVs)) and decreased RBC survival. ${ }^{1}$ The haematological findings often provide the impetus for assessing vitamin B12 and folate status in the patient when the clinical picture is uncertain. However, haematological changes are usually a sign of established deficiency ${ }^{1}$ which limits its diagnostic use. Patients should potentially be assessed for deficiency at an earlier stage to prevent complications. ${ }^{3}$ Microcytosis may be a feature if patients have coexisting iron deficiency, chronic disease or thalassaemia, ${ }^{1}$ and these patients are at risk of not being investigated for deficiency.

\section{Metabolites}

Deficiencies in vitamin B12 result in accumulations of methylmalonyl-coenzyme A and its product methylmalonic acid (MMA). ${ }^{6,8}$ MMA measurement is used as a specific diagnostic test for vitamin B12 deficiency. ${ }^{1,5}$ In addition, both folate and vitamin B12 pathway defects result in an elevation of homocysteine levels. ${ }^{9} \quad$ Elevated levels of MMA and/or homocysteine are evident in very early vitamin B12 and folate deficiency before measurable decreases in serum vitamin B12 and folate levels are present. ${ }^{5,10}$ Their measurements are thus useful in patients with no anaemia or macrocytosis. ${ }^{10}$

\section{Investigation}

Serum vitamin B12 and folate levels are routinely performed as the first-line test of vitamin B12 status internationally, despite MMA and homocysteine measurements being more sensitive diagnostic markers of deficiency. ${ }^{11}$ These second line tests are not readily available and are limited to a few specialised laboratories. ${ }^{1,11}$ Akin to international standards (11), serum B12 and folate levels are the first line of investigating deficiencies at Tygerberg Academic Hospital (TAH). ${ }^{12}$ Local recommendations state that in populations where folate food fortification occurs, such as in South Africa, optimal assessment of vitamin status should include both serum and red blood cell (RBC) folate assays, as well as serum vitamin $\mathrm{B} 12$ measurement. ${ }^{7}$ RBC folate is however no longer performed at Tygerberg Academic Hospital (TAH) within the National Health Laboratory Service (NHLS) in South Africa due to the numerous analytical problems with this assay. ${ }^{11}$

In developed countries, folate testing is often performed in the setting of haematological changes, alcoholism, malabsorption, in certain haematological diseases and in chemotherapy patients. ${ }^{7}$ In developing countries however, it is commonly associated with primary health care problems such as HIV infection, poor nutritional intake or the increased demands of pregnancy. ${ }^{7}$ Similarly studies on vitamin B12 and folate in South Africa are based on small 
target populations identified to be at increased risk of nutritional deficiency. ${ }^{13-16}$ This study however, looks at patterns of testing in patients in a tertiary referral hospital. It focuses on patients who have had routine FBC testing displaying the abnormal haematological parameters of macrocytosis and/or anaemia to determine whether they have been appropriately screened for such advanced vitamin $\mathrm{B} 12$ or folate deficiency.

\section{METHODS}

This study was a retrospective audit of laboratory tests for vitamin B12 and folate deficiency in patients with (a) macrocytic anaemia, (b) isolated macrocytosis and (c) normocytic anaemia at TAH, Western Cape Province, South Africa. TAH is a large tertiary referral hospital serving approximately half of the population of the Western Cape Province of 5 million people, as well as neighbouring provinces in certain instances. The NHLS at TAH performs approximately 6300 serum vitamin B12 and 3430 folate assays on average per annum. The study was approved by the Ethics Committee of the University of Stellenbosch (reference number S15/07/138).

Data was extracted from the laboratory information system (DisaLab), from 1 January 2015 to 30 June 2015. Study inclusion criteria were all patients greater and equal to 18 years of age who had either (a) macrocytic anaemia
(MCV $>100 \mathrm{fl} ; \mathrm{Hb}<13 \mathrm{~g} / \mathrm{dL}$ in males and $<12 \mathrm{~g}$ / $\mathrm{dL}$ in females), (b) isolated macrocytosis (MCV >100fl; $\mathrm{Hb} 13 \mathrm{~g} / \mathrm{dL}-17 \mathrm{~g} / \mathrm{dL}$ in males and $12 \mathrm{~g} / \mathrm{dL}-15 \mathrm{~g} / \mathrm{dL}$ in females) or (c) normocytic anaemia (MCV 79.1 - 99.9fl; $\mathrm{Hb}$ $<13 \mathrm{~g} / \mathrm{dL}$ in males and $<12 \mathrm{~g} / \mathrm{dL}$ in females). A cut-off value of $100 \mathrm{fl}$ for the MCV was used to identify macrocytosis. Samples that were rejected due to haemolysis or insufficiency $(n=11)$ were included in the study as these patients were screened for vitamin status despite the outcome of the tests. Several conditions are known to cause high MCVs without abnormalities in vitamin B12 and folate metabolism. These include myelodysplastic syndromes, aplastic anaemia, post-haemorrhagic anaemia, acute haemolytic disease, acute liver disease, obstructive jaundice and certain antiretroviral treatment (e.g. zidovudine or stavudine) or cytotoxic drugs (e.g. hydroxyurea). ${ }^{1,8,17,18}$ These patients were excluded from the study.

All laboratory records of patients with isolated macrocytosis who had vitamin B12 or folate testing performed were further studied to assess whether they were being followed-up for deficiency from the previous year (2014) or whether these were new cases for investigation. Laboratory records for 2014 were also reviewed in a randomly selected group of isolated anaemia patients $(19.3 \%$; $\mathrm{n}=100$ ). Patients not included in the above groups who had vitamin B12 and folate testing

Table 1: Number of patients tested for vitamin B12 and/or folate in the macrocytic anaemia, isolated macrocytosis

\begin{tabular}{|c|c|c|c|}
\hline & \multicolumn{3}{|c|}{ Groups Assessed for Vitamin B12 and/or Folate Testing $(n=10,308)$} \\
\hline & $\begin{array}{l}\text { Macrocytic Anaemia } \\
(\mathrm{MCV}>100 \mathrm{fl} ; \mathrm{Hb}<13 \mathrm{~g} / \\
\mathrm{dL} \text { in males and }<12 \mathrm{~g} / \mathrm{dL} \\
\text { in females })\end{array}$ & $\begin{array}{l}\text { Isolated Macrocytosis } \\
(\mathrm{MCV}>100 \mathrm{fl} ; \mathrm{Hb} 13-17 \mathrm{~g} / \\
\mathrm{dL} \text { in males and } 12-15 \mathrm{~g} / \mathrm{dL} \\
\text { in females) }\end{array}$ & $\begin{array}{l}\text { Normocytic Anaemia } \\
(\mathrm{MCV} 79.1-99.9 \mathrm{fl} ; \\
\mathrm{Hb}<13 \mathrm{~g} / \mathrm{dL} \text { in males and } \\
<12 \mathrm{~g} / \mathrm{dL} \text { in females) }\end{array}$ \\
\hline $\begin{array}{l}\text { Total number of patients } \\
(\mathrm{n} ; \%)\end{array}$ & $1,131(11.0 \%)$ & $1,265(12.3 \%)$ & $7,912(76.8 \%)$ \\
\hline $\begin{array}{l}\text { Patients tested for vitamin } \\
\text { B12 and/or folate (n;\%) }\end{array}$ & $183(16.2 \%)$ & $99(7.8 \%)$ & $518(6.5 \%)$ \\
\hline
\end{tabular}


without any recorded FBC results, as well as patients with a normal MCV (79.1 - 99.9fl) and $\mathrm{Hb}$ or microcytosis $(\mathrm{MCV}<79.1 \mathrm{fl})$, were also assessed for the cause of testing $(n=205)$. Data were analysed using Microsoft ${ }^{\circledR}$ Excel ${ }^{\circledR}$ (Microsoft Inc., Redmond, WA).

At TAH laboratory, haemoglobin and mean cell volume assays are performed through flow cytometry technique (Siemens ADVIA 2120 analyser, Siemens, Erlangen, Germany) and vitamin B12, folate and homocysteine assays are performed through immunoassay with a chemiluminescent endpoint (Siemens ADVIA Centaur analyser). TAH NHLS is accredited by the South African National Accreditation System (SANAS) and belongs to internal and external proficiency testing schemes, namely Biorad ${ }^{\circledR}$ for vitamin B12, folate and homocysteine and the Royal College of Pathologists of Australia (RCPA) for $\mathrm{Hb}$ and MCV.

\section{RESULTS}

A total of 10,308 patients were identified from 43,216 full blood count results fulfilling the inclusion criteria. Of these patients, $11.0 \%$ had macrocytic anaemia $(n=1,131), 12.3 \%$ had isolated macrocytosis $(n=1,265)$ and $76.8 \%$ had normocytic anaemia $(n=7,912)$. In total, $16.2 \%$ in the macrocytic anaemia group $(\mathrm{n}=183), 7.8 \%$ in the isolated macrocytosis group ( $\mathrm{n}=99)$ and $6.5 \%$ in the normocytic anaemia group $(n=518)$ were tested for either vitamin B12 or folate deficiency or both. Results are summarised in Table 1.

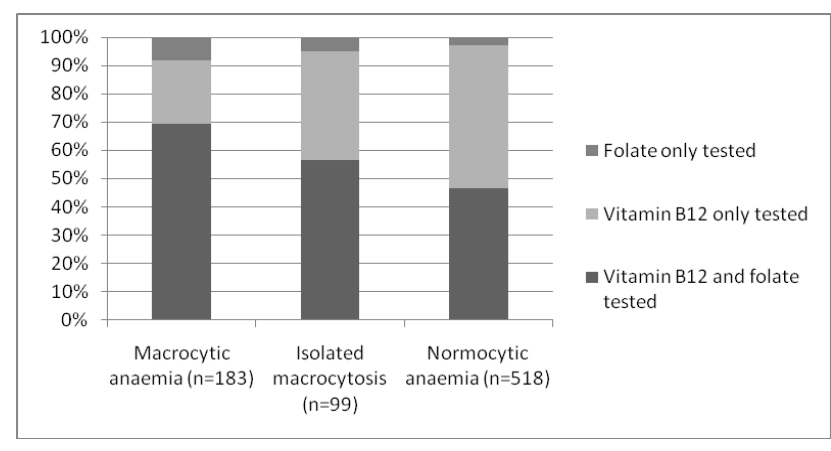

Figure 1: Proportion of patients tested for vita$\min \mathrm{B} 12 \mathrm{and} /$ or folate in the macrocytic anaemia, isolated macrocytosis and normocytic anaemia groups.
In the isolated macrocytosis group, $16.2 \%$ $(n=16 / 99)$ of patients who were tested for vitamin B12 or folate were previously tested for vitamin B12 and/or folate in 2014. These patients were follow-up patients from the renal or oncology units at TAH where routine vitamin $\mathrm{B} 12$ and folate testing takes place. Of the randomly selected group of patients $(n=100)$ from the normocytic anaemia group, $20 \%(n=20)$ were follow-up patients. These patients were also predominantly from the renal unit $(65 \% ; n=13)$ where dialysis and renal failure patients are routinely screened.

A further 205 vitamin B12 and folate tests, not accounted for in the above groups, were requested during the six month period. These tests were performed in patients who had a normal haemoglobin and MCV or in patients who were microcytic, as well as in patients who had no recorded FBC test during the period. The laboratory records were subsequently followed up to assess the reason for their vitamin B12 and folate testing, the results of which are illustrated in table 2.

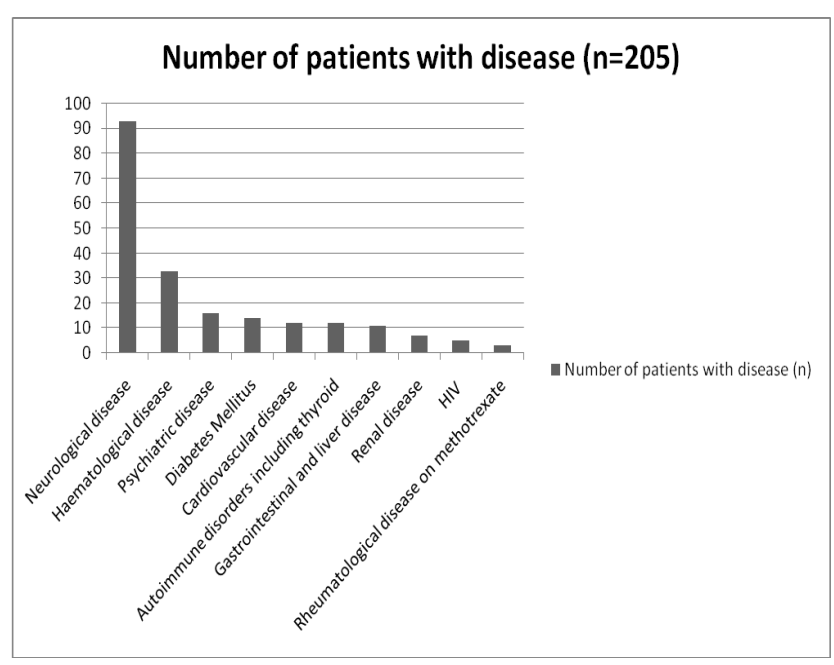

Figure 2: Reasons for vitamin B12 and folate testing in patients without macrocytosis and/or anaemia and in patients with no full blood count tests.

The largest proportion of patients had neurological, haematological or psychiatric diagnoses. The remainder of the patients were from various medical specialities with conditions associated with vitamin $\mathrm{B} 12$ or folate deficiency or drugs known to affect either vitamin B12 or folate metabolism. ${ }^{1}$ 
The average rates of deficiency of vitamin B12 and folate (all groups) were $3.7 \%$ and $8.7 \%$ respectively. Only $0.3 \%$ of patients tested were deficient for both vitamins in all groups. Of all the groups, only one patient had a serum homocysteine level requested in relation to cardiovascular disease rather than for assessment of vitamin status. No requests for the referral of MMA assays were found for the entire period.

Of the samples tested for vitamin B12 and folate, $1.6 \%(\mathrm{n}=3)$ in the isolated macrocytosis group, and $1 \% \quad(n=5)$ in the normocytic anaemia group were haemolysed and thus vitamin B12 and folate assays could not be performed. No repeat testing was performed in these patients.

\section{DISCUSSION}

Vitamin B12 and folate deficiencies are common, easily treated conditions. In a small study $(n=80)$ performed in rural women of reproductive age in South Africa, rates of vitamin B12 deficiency were shown to be between $6-11 \%$, and approximately $27 \%$ for folate deficiency. ${ }^{13}$ As these results were not confirmed by metabolite testing, true rates of deficiency may thus be higher due to the low sensitivity and specificity of the assays used. ${ }^{1,6,11}$ According to the British Committee for Standards in Haematology, the clinical assessment of the patient is still the most important aid to interpreting test results of vitamin B12 status as there is no 'gold standard' test to assess deficiency. ${ }^{11}$ Haematological parameters are thought to be less useful in identifying such patients. In early deficiency, haemoglobin $(\mathrm{Hb})$ and mean cell volumes (MCVs) may be normal. As deficiency worsens, there is a progressive increase in $\mathrm{MCV}$, and later, a decrease in $\mathrm{Hb} .{ }^{1}$ In a study by Metz et al., significant changes in MCVs of RBCs were not found until vitamin B12 concentrations were below 200ng/L (normal range 211-911 $\mathrm{ng} / \mathrm{L}$ ). ${ }^{19}$ Approximately $20-40 \%$ of all vitamin B12 deficient patients have normal $\mathrm{Hb}$ levels, normal MCVsor have normal levels of both. ${ }^{3,20}$ -22 However, without a clear clinical presentation, the threshold for assessing patients' vitamin status should be low if they have deranged haematological parameters as seen in the study groups. This may be the only feasible option where resources are limited and routine FBCs are available for analysis.

The aim of our study was thus to assess whether patients with deranged haematological parameters were assessed for vitamin B12 or folate deficiency. We observed that patients with macrocytic anaemia were more frequently tested for vitamin B12 and folate and thus were more commonly associated with vitamin B12 and/or folate deficiency compared to patients with isolated macrocytosis and normocytic anaemia. However, despite the large number of patients presenting with macrocytic anaemia $(\mathrm{n}=1,131)$, only $16.2 \%$ were tested for vitamin B12 and/or folate deficiency $(\mathrm{n}=183)$.

Only $7.7 \%$ of patients with isolated macrocytosis and $6.5 \%$ of patients with normocytic anaemia were tested for vitamin B12 and/or folate. The data was revisited to understand reasons for testing in these two groups. In a random sample it was noted that on average, almost a fifth of the patients who had been tested during the study period had been tested previously as part of a renal or oncological work-up. The inclusion of these patients receiving follow-up testing to the isolated macrocytosis and normocytic anaemia groups further decreases the number of new patients identified and screened for deficiency based on haematological parameters. The significance of a high MCV or anaemia as a sign of advanced deficiency appears to be underestimated. It is clear from the data that the assessment of patients with the presence of either haematological variables (macrocytosis or anaemia) can be improved upon.

Normocytic anaemia may perhaps more often be associated with other causes, such as anaemia of chronic disease. ${ }^{23}$ Should this be the case, chronic diseases and chronic medication use in itself warrants vitamin B12 and folate status assessment due to likely changes in intake, absorption, metabolism, storage or excretion of the vitamins. The coexistence of iron deficiency with vitamin B12 or folate deficiency, resulting in normocytosis, ${ }^{1}$ should be considered especially in patients with overall poor nutritional status. Few patients in all groups were tested for folate 
in isolation $(2.7-8.2 \%)$, and overall vitamin B12 testing is more common. Whether vitamin B12 testing is influenced by the clinical picture of the patient for example neuropathology which is more specific to B12 deficiency, or whether clinicians understand the importance of testing for the two vitamins simultaneously in suspected folate deficiency remains unclear but could possibly account for the higher rates of vitamin B12 assay requests.

We observed that vitamin B12 and folate levels were routinely measured in patients with normal FBCs or microcytosis, or patients who had no recorded FBCs. This is likely due to the recognised effects of the illness or treatment on vitamin metabolism, or the patients' clinical presentation. The Neurology unit for example, routinely screens all patients with neuropathy or myelopathy for vitamin B12 and folate deficiency irrespective of their haematological parameters. These additional findings indicate that some medical specialities do test for vitamin B12 and folate routinely and are aware of the possible pitfalls of relying on FBC findings only.

Few patients tested appeared to be deficient for either vitamin B12 (3.7\%) or folate $(8.7 \%)$ respectively in all groups. However, in this study deficiency cannot be confirmed nor excluded based on observing the results of a single serum vitamin B12 or folate level alone. In addition, second line tests, such as homocysteine and MMA known to be more useful in the definitive diagnosis of vitamin B12 and folate deficiencies ${ }^{1,11}$ were not requested. In the groups, only one patient had a homocysteine level requested in relation to cardiovascular disease. This suggests either a lack of awareness of the role of metabolite testing, or lack of awareness that it is offered at our laboratory. The purpose and benefits of metabolite testing, in particular homocysteine assays, need to be carefully outlined as it may promote early assessment and the prevention of complications. The cost of a homocysteine assay in South Africa is comparable to a serum vitamin $\mathrm{B} 12$ or a folate assay (+/- US $\$ 9$ vs US\$7.4), and is thus an affordable way of minimising the costly complications of vitamin B12 and folate deficiency. The limitations of homocysteine testing however need to be taken into account. Its specificity for vitamin B12 and folate deficiency is decreased in renal failure, alcohol abuse, vitamin B6 deficiency, hypothyroidism and in certain medication where homocysteine levels may be elevated. ${ }^{1}$ MMA assays are not offered at TAH laboratory, but when requested, are referred to a nearby laboratory. The MMA assay however, is almost nine times the cost of an assay for vitamin B12 or folate.

Studies indicate that using combined markers for determining vitamin B12 status is more effective at diagnosing subclinical deficiency and for distinguishing patients who are at risk of deficiency than using one marker alone. ${ }^{24}$ This study has indicated the need for the development of clear guidelines for clinicians, outlining the process of homocysteine testing in addition to routine serum vitamin B12 and folate levels when suspected patients present with normal vitamin B12 or folate levels. Vitamin B12 absorption and transport is dependent on a group of proteins called the transcobalamins I, II and III. ${ }^{1}$ Transcobalamin II, the 'active' fraction of vitamin B12, may be a more specific determinant of serum vitamin B12 deficiency as it shows better performance than serum vitamin B12. ${ }^{11}$ Testing is very rarely performed but may become the norm in future diagnostic laboratories. Its discussion is beyond the scope of this study.

Clinicians should also be informed that haemolysis of the collected sample will result in sample rejection by the laboratory and thus phlebotomy technique is of importance during sample collection.

This audit was performed at a tertiary hospital where metabolite testing is available and the vitamin B12 and folate status of patients can be monitored. It was however limited to patients seen at TAH and is not representative of the larger South African population. In the isolated macrocytosis group $(n=1194)$, it could not be determined from the laboratory records whether the $0.4 \%$ of patients with chronic myeloid leukaemia untested for vitamin B12 and folate $(n=5)$ were on treatment that could cause elevated MCVs. This may subsequently explain the lack of screening for vitamin B12 and folate deficiency in these patients. The clinical records of the patients were not studied and more detailed information pertaining to the diagnosis, management and pharmacotherapy 


\section{CONCLUSION}

Patients with a strong clinical suspicion of to have macrocytic anaemia, isolated macrocytosis or normocytic anaemia should be assessed for vitamin B12 and folate deficien-cies. Under-investigation is a common occurrence and needs to be addressed by educating clinicians on the need for more frequent assessment of vitamin B12 and folate status, as well as metabolite testing in selected patients. Deficient patients can be thus be promptly identified and managed accordingly, avoiding late and costly treatment of severe and debilitating complica-tions.

\section{CONFLICT OF INTEREST}

\section{None Declared}

\section{REFERENCES}

1. Wickramasinge SL. Diagnosis of Megaloblastic anamias. Blood Reviews. 2006; 20:299-318.

2. Sobczynska-Malefora A, Gorska R, Pelisser M, et al. An audit of holotranscobalamin ("Active B12") and methyl-malonic acid assays for the assessment of vitamin B12 status: Application in a mixed patient population. Clinical Biochemistry.2014;47:82-86.

3. Lindenbaum J, Healton EB, Savage DG, et al. Neuropsychiatric disorders caused by cobalamin deficiency in the absence or anaemia or microcytosis. New England Jour-nal of Medicine.988; 318:1720-1728.

4. Solomon L. Disorders of cobalamin (Vitamin B12) me-tabolism: Emerging concepts in pathophysiology, diagno-sis and treatment. Blood Reviews. 2007; 21: 113-130 .

5. Klee G. Cobalamin and Folate Evaluation: Measurement of Methylmalonic Acid and Homocysteine vs Vitamin B12 and Folate. Clinical Chemistry. 2000; 46(8): 1277-1283 .

6. Snow CF. Laboratory Diagnosis of Vitamin B12 and Folate Deficiency. American Medical Association. 1999; 159: 1289-1298 .

7. Metz J. Haematological implications of folate food fortifi-cation. South African Medical Journal. 2013; 103 (12): 978-981 .

8. Chanarin I, Metz J. Diagnosis of cobalamin deficiency: the old and the new. British Journal of Haematology. 1997; 97: 695-700 .

9. Perry DJ. Hyperhomocysteinaemia. Bailliere's Clinical Haematology. 1999; 12 ( 3 ) : 451-477

10. Green R. Metabolite assays in cobalamin and folate defi-ciency. Bailiere's Clinical Haematology. 1995; 8(3): 533-566 .

11. Devalia D, Hamilton MS, Molloy AM. Guidelines for the diagnosis and treatment of cobalamin and folate disor-ders. British Journal of Haematology. 2014; 166: 496-513.
12. NHLS Standard Operating Procedures for ADVIA Centaur Immunoassay System. 2015.

13. Modjadji SEP, Alberts M, Mamabolo RL. Folate and iron status of non-pregnant South African rural women of childbearing age, before and after fortification of food. South African Journal of Clinical Nutrition. 2007; 20(3): 89 -93

14. Charlton KE, Kruger M, Labadarios D, et al. Iron, folate and vitamin B12 status of an elderly South African popu-lation. European Journal of Clinical Nutrition. 1997; 51: 424-430

15. Baynes RD, Meriwether WD, Bothwell TH, et al. Iron and folate status of pregnant black women in Gazankulu. South African Medical Journal. 1986; 70:148-151

16. Ubbink JB, Christianson A, Bester MJ, et al. Folate status, homocysteine metabolism and methylene tetrahydro- folate reductase genotype in rural South African blacks with a history of pregnancy complicated by neural tube defects. Metabolism.1999; 48 : 269-274

17. Aslinia F, Mazza JJ, Yale SH. Megaloblastic anaemia and other causes of macrocytosis. Clinical Medicine and Re-search. 2006; 4(3): 236-241.

18. Romanelli F, Empey K, Pomeroy C. Macrocytosis as an indicator of medication (zidovudine) adherence in pa-tients with HIV infection. AIDS Patient Care and STDs. 2002; 16(9): 405-411

19. Metz J, Bell AH, Flicker L, et al. The significance of sub-normal serum vitamin B12 concentration in older people: a case study. Journal of American Geriatric Society. 1996; 44: 1355-1361.

20. Carmel R. Pernicious Anaemia. The expected findings of very low serum cobalamin levels, anaemia and macrocy-tosis are often lacking. Archives of Internal Medicine. 1988; 148: 1712-1714

21. Stabler SP, Allen RH, Savage DG, et al. Clinical spectrum and diagnosis of cobalamin deficiency. Blood. 1990; 76: 871-881

22. Thompson WG, Cassino C, Babitz L, et al. Hyperseg- mented neutrophils and vitamin B12 deficiency. Ac-taHaematologica. 1989; 81: 186191

23. Krantz S. Pathogenesis and treatment of anaemia of chronic disease. American Journal of the Medical Sci-ences. 1994; 307(5): 353-359

24. 24. Fedosov SN, Brito A, Miller JW, et al. Combined indicator of vitamin B12 status: modification for missing bio-markers and folate status and recommendations for re-vised cut-points. Clinical Chemistry Laboratory Medicine. 2015; 53(8): 12151225 